\title{
Asymmetric Catalysis via Cyclic, Aliphatic Oxocarbenium lons
}

\author{
Sunggi Lee, Philip S. J. Kaib, and Benjamin List* \\ Max-Planck-Institut für Kohlenforschung, Kaiser-Wilhelm-Platz 1, 45470 Mülheim an der Ruhr, Germany
}

\begin{abstract}
A direct enantioselective synthesis of substituted oxygen heterocycles from lactol acetates and enolsilanes has been realized using a highly reactive and confined imidodiphosphorimidate (IDPi) catalyst. Various chiral oxygen heterocycles, including tetrahydrofurans, tetrahydropyrans, oxepanes, chromans, and dihydrobenzofurans were obtained in excellent enantioselectivities by reacting the corresponding lactol acetates with divers enol silanes. Mechanistic studies suggest the reaction to proceed via a non-stabilized, aliphatic, cyclic oxocarbenium ion intermediate paired with the confined chiral counteranion.
\end{abstract}

Oxocarbenium ions are key intermediates in enzymatic glycosidations but also in a variety of synthetic transformations. ${ }^{1}$ The stereocontrol of such reactions is challenging and catalytic enantioselective approaches have only recently been described. ${ }^{2}$ Asymmetric nucleophilic substitutions via non-aromatic, cyclic oxocarbenium ions are particularly difficult to control as these cations neither display Lewis basic sites nor large $\pi$-surfaces for substrate-catalyst interactions. We show here that silylium-based, asymmetric counteranion directed Lewis acid catalysis provides a general solution to this challenge; various lactol acetates react with enol silanes in the presence of a highly active and confined pre-Lewis acid organocatalyst to furnish substituted oxygen heterocycles in high enantioselectivity.

Despite the frequent appearance of saturated oxygen heterocycles in drugs and natural products, ${ }^{3}$ general methods for their enantioselective synthesis are relatively rare. ${ }^{4-11}$ One straight forward approach is the direct addition of nucleophiles to in situ generated cyclic oxocarbenium ions. In pioneering studies, Braun et al. showed that a chiral titanium complex enables the dynamic kinetic asymmetric nucleophilic substitution of allyltrimethylsilane with silyl ethers and acetals. ${ }^{2 a}$ However, high enantioselectivity was only achieved with aromatic substrates. Recently, the Jacobsen group reported enantioselective substitution reactions of silyl ketene acetals with aromatic glycosyl chlorides using anion binding catalysis. ${ }^{2 \mathrm{~b}, 2 \mathrm{r}-\mathrm{t}}$ Chiral 1 -substituted isochromanes and related products were also obtained via oxidative enamine catalysis, via alkynylation using a chiral copper catalyst combined with stoichiometric Lewis acids, via chiral silver salt catalyzed reductions, and via nucleophilic addition reactions to pyrylium salts using different catalysts. ${ }^{2 \mathrm{c}-0}$ Most recently, the Mattson group developed a silandiol catalyzed chromenone functionalization $^{2 p}$ and the Toste group utilized anionic phase transfer catalysis for the formation of 2,4 diarylbenzopyrans. ${ }^{2 q}$

All these examples are limited to the use of resonance stabilized oxocarbenium ions within six membered ring systems. In contrast, the enantioselective formation of tetrahydrofurans or tetrahydropyrans via nucleophilic addition reactions to aliphatic, cyclic oxocarbenium ions remained as a long-standing challenge. Their high electrophilicity often leads to catalyst degradation and their small size and the absence of further functionalization makes enantiodifferentiation difficult to accomplish. We have recently introduced confined imidodiphosphoric acid catalysts (IDPs) that catalyze cyclization reactions via aliphatic oxocarbenium ions. ${ }^{12}$ However, intermolecular reactions proceeding via unmodified, aliphatic, cyclic oxocarbenium ions could not be accomplished with these catalysts. We envisioned that our newly developed imidodiphosphorimidates (IDPi) could perhaps provide a solution to this significant problem. ${ }^{13}$ Their acidity is higher than that of IDPs and even of disulfonimides (DSIs), enabling silylium-based asymmetric counteranion directed catalysis (Si-ACDC). ${ }^{14,15}$ In addition, their highly stabilized counteranions should support the generation of ion pairs with strongly electrophilic cations. Finally, the confined active site should allow an efficient enantiodiscrimination of sterically and electronically unbiased cationic intermediates. Based on these considerations, a plausible catalytic cycle can be envisioned (eq. 1), which is initiated with the in situ generation of the silylium ion pair catalyst from the strongly Brønsted acidic precatalyst $\mathrm{HX}^{\star}$ and a silylated nucleophile (TBS-Nu).

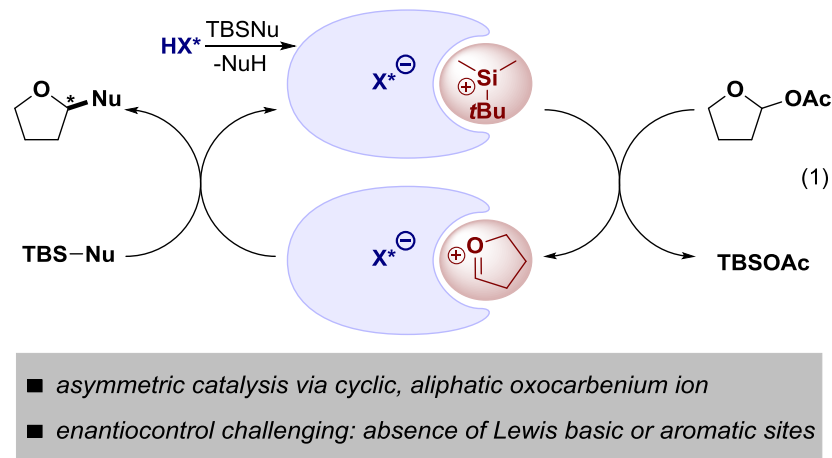


The cyclic oxocarbenium ion is subsequently generated via acetate abstraction from a lactol acetate by the silicon Lewis acid. $^{16}$ In the following nucleophilic addition of the silylated nucleophile to the oxocarbenium ion, the counteranion directs the enantiodifferentiation leading to formation of the product and the regeneration of the silylium Lewis acid catalyst.

To test this design, we explored the reaction of different oxocarbenium ion precursors 1 with ketene acetal $2 \mathbf{a}$ in the presence of a variety of acidic catalysts previously developed in our group (Table 1). Chiral disulfonimide catalyst 4 enabled full conversion to the desired product $3 a$ within $4 \mathrm{~h}$, but without any enantioselectivity (entry 1). In contrast, imidodiphosphate catalyst $\mathbf{5}$ gave no conversion, likely due to its insufficient acidity. Remarkably however, IDPi catalyst

Table 1. Reaction Development ${ }^{\mathrm{a}}$
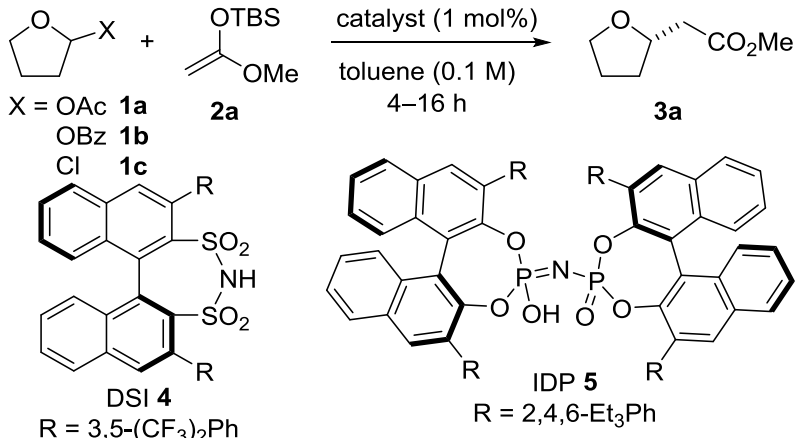

$\mathrm{R}=3,5-\left(\mathrm{CF}_{3}\right)_{2} \mathrm{Ph}$

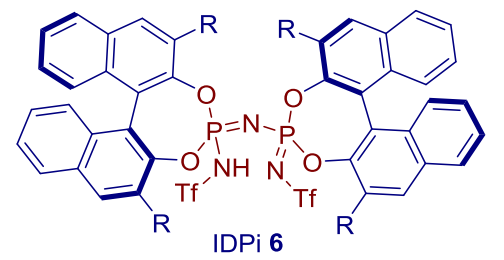

$\mathrm{R}=\mathrm{Ph} \quad 6 \mathrm{a}$ 2-Naph 6b 4-Me-Ph 6c 4-t-Bu-Ph 6d

\begin{tabular}{llllll}
\hline entry & substrate & catalyst & Temp & conv. & er \\
\hline $1^{\mathrm{c}, \mathrm{d}}$ & $\mathbf{1 a}$ & $\mathbf{4}$ & r.t. & $>95$ & $50: 50$ \\
$2^{\mathrm{c}, \mathrm{d}}$ & $\mathbf{1 a}$ & $\mathbf{5}$ & r.t. & $<10$ & $\mathrm{ND}$ \\
$3^{\mathrm{c}}$ & $\mathbf{1 a}$ & $\mathbf{6 a}$ & r.t. & $>95$ & $59.5: 40.5$ \\
4 & $\mathbf{1 a}$ & $\mathbf{6 a}$ & r.t. & $>95$ & $61.5: 18.5$ \\
5 & $\mathbf{1 a}$ & $\mathbf{6 b}$ & $-20{ }^{\circ} \mathrm{C}$ & $>95$ & $60: 40$ \\
6 & $\mathbf{1 a}$ & $\mathbf{6 c}$ & $-20^{\circ} \mathrm{C}$ & $>95$ & $84: 16$ \\
7 & $\mathbf{1 a}$ & $\mathbf{6 d}$ & $-20^{\circ} \mathrm{C}$ & $>95$ & $94: 6$ \\
8 & $\mathbf{1 b}$ & $\mathbf{6 d}$ & $-20^{\circ} \mathrm{C}$ & $>95$ & $93: 7$ \\
9 & $\mathbf{1 c}$ & $\mathbf{6 d}$ & $-20{ }^{\circ} \mathrm{C}$ & $>95^{\mathrm{e}}$ & $87: 13$ \\
10 & $\mathbf{1 a}$ & $\mathbf{6 d}$ & $-78^{\circ} \mathrm{C}$ & $>95$ & $97: 3$ \\
$11^{f}$ & $\mathbf{1 a}$ & $\mathbf{6 d}$ & $-78^{\circ} \mathrm{C}$ & $>95$ & $98: 2$ \\
$12^{\mathrm{f}, \mathrm{g}}$ & $\mathbf{1 a}$ & $\mathbf{6 d}$ & $-78^{\circ} \mathrm{C}$ & $>95(88)^{\mathrm{h}}$ & $98: 2$ \\
\hline
\end{tabular}

${ }^{a}$ Unless otherwise indicated, reactions were conducted on 0.05 mmol scale with 3.0 equiv. of $2 a$ and $1.0 \mathrm{~mol} \%$ of catalyst in $0.5 \mathrm{~mL}$ of toluene. Er was determined by GC. ${ }^{b}$ Conversions were determined by NMR with an internal standard. ${ }^{\mathrm{C}}$ Reaction was carried out in $\mathrm{CH}_{2} \mathrm{Cl}_{2}{ }^{d} 5$ mol\% catalyst. ${ }^{e} 19 \%$ of product was observed in crude NMR ${ }^{\dagger} 1.2$ equiv. nucleophile ${ }^{g} 1.0 \mathrm{mmol}$ scale, $0.1 \mathrm{~mol} \%$ catalyst hisolated yield; $\mathrm{ND}=$ not determined 6a efficiently promoted the reaction with promising enantioselectivity (entries 3,4). The corresponding 2-naphtyl substituted catalyst $\mathbf{6 b}$, previously used in the asymmetric Hosomi-Sakurai allylation of aldehydes, showed no improvement in enantioselectivity (entry 5 ). We found that para-substituents at the 3,3'-phenyl-groups of the BINOLbackbone strongly affect the enantioselectivity. Stepwise er increase was observed by varying from the small methyl to the bulky tert-butyl groups, affording up to $94: 6$ er at $-20^{\circ} \mathrm{C}$ in toluene (entries 6,7 ). Switching from acetate $1 \mathrm{a}$ to benzoate $\mathbf{1 b}$ did not influence reactivity or selectivity (entry 8 ) and 2-chlorotetrahydrofuran 1c gave only $19 \%$ of the product and reduced enantioselectivity. The selectivity was further increased to $97: 3$ er by lowering the temperature to $-78{ }^{\circ} \mathrm{C}$ (entry 10). Furthermore, the amount of nucleophile could be reduced to 1.2 equiv. without affecting enantioselectivity or conversion (entry 11). Under the optimal condition, as little as $0.1 \mathrm{~mol} \%$ of catalyst $6 \mathbf{d}$ was sufficient to furnish tetrahydrofuran $\mathbf{3 a}$ in $88 \%$ yield after 16 hours (entry

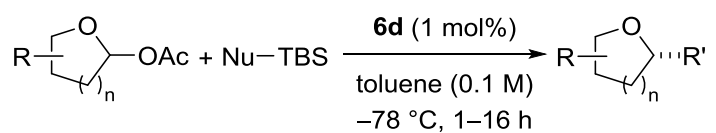

12). It is noteworthy that product $3 \mathbf{a}$ is the staring material of several syntheses of biologically active materials but there are only a few preparative procedures for its synthesis, using enzymatic kinetic resolutions. ${ }^{17}$

With the optimal condition in hand, we next investigated the reaction scope (Table 2). Various nucleophiles and electrophiles can be utilized in our reaction. The size of the nucleophile does not seem to influence selectivity and reactivity much (entries 1-3). In addition to silyl ketene acetals, the silyl enol ether derived from acetophenone (2d) was also applicable, although with slightly lower enantioselectivity (entry 4). A conjugated silyl enol ether gave product $3 e$ in high enantioselectivity (entry 5). Extended nucleophiles such as vinylogous ketene acetal $\mathbf{2} \mathbf{f}$ and dioxinone-derived silyloxydiene $\mathbf{2 g}$ reacted at the $\mathrm{y}$-position, furnishing the corresponding heterocycles with more than 95:5 er and in moderate to excellent yields (entries 6,7$)$. Tetrahydropyran and oxepane products $\mathbf{3} \mathbf{h}$ and $\mathbf{3 i}$ could be obtained under identical conditions with high enantiomeric ratios from the corresponding 6- and 7-membered lactol acetates (entries $8,9)$. In addition to simple aliphatic substrates, aromatic substrates possessing chroman and benzodihydrofuran moieties could also be converted under our reaction conditions, but required longer reaction times and higher concentration. Full conversion to products $\mathbf{3} \mathbf{j}$ and $\mathbf{3 k}$ with excellent and good enantioselectivity was observed after seven and four days respectively (entries 10,11). In contrast, the reaction with 1 -acetoxyisochroman $1 \mathrm{~h}$ was completed within 10 min and gave product $\mathbf{3}$ I in high yield, but with only moderate enantioselectivity (entry 12 ).

Remarkably, when 2,4-bis(acetoxy)-tetrahydrofuran 1i was subjected to reaction conditions using 2.4 equiv. of nucleophile $\mathbf{2} \mathbf{b}$, the entire mixture of stereoisomers (meso-1i, $S, S$ $1 \mathrm{i}$, and $R, R-\mathbf{1 i})$ was converted into a single major product stereoisomer $(\mathrm{Eq} 2)$. The initial 1.5:1 racemic trans to cis ratio of substrate $1 \mathbf{i}$ gave a 15:1 trans to cis ratio in product $3 \mathrm{~m}$, which was obtained with superb enantiomeric ratio $(>99: 1)$ in a diasteroconvergent and enantioconvergent kinetic resolution. We were also able to utilize $\alpha$-branched nuclophiles. For example, 2-(trimethylsiloxy)furan $(\mathbf{2 h})$ re- 
acted with lactol acetate 1a to give lactone product syn-3n with excellent er and moderate $\mathrm{dr}(\mathrm{Eq} 3) .{ }^{18}$

Table 2. Substrate Scope ${ }^{a}$

\begin{tabular}{|c|c|c|c|c|}
\hline entry & product & $\mathrm{Nu}-\mathrm{TBS}$ & $\begin{array}{c}\text { yield } \\
(\%)\end{array}$ & er \\
\hline $1^{\mathrm{b}}$ & $3 \mathbf{a}$ & $\begin{array}{l}\text { TBS } \\
\text { OMe } \\
\mathbf{2 a}\end{array}$ & 88 & $98: 2$ \\
\hline 2 & 3b & 2b & 94 & $97: 3$ \\
\hline 3 & $3 c$ & $\begin{array}{l}\mathrm{O}-\mathrm{c}-\mathrm{H} \\
\mathbf{2 c}\end{array}$ & 95 & $96: 4$ \\
\hline $4^{c}$ & 3d & $2 d$ & 97 & $89.5: 10.5$ \\
\hline 5 & $3 \mathbf{e}$ & $2 e$ & 74 & $95: 5$ \\
\hline 6 & $3 \mathbf{f f}$ & $2 f$ & 70 & $96.5: 3.5$ \\
\hline 7 & $3 \mathrm{~g}$ & $2 \mathrm{~g}$ & 90 & $96.5: 3.5$ \\
\hline $8^{c}$ & $3 h$ & $2 b$ & 80 & $96.5: 3.5$ \\
\hline 9 & $3 \mathbf{i}$ & $2 b$ & 91 & $97: 3$ \\
\hline $10^{\mathrm{d}, \mathrm{e}}$ & $3 \mathbf{j}$ & $2 b$ & 43 & $98.5: 1.5$ \\
\hline $11^{\mathrm{d}, \mathrm{f}}$ & $3 \mathbf{k}$ & $2 b$ & 96 & $94.5: 5.5$ \\
\hline $12^{\mathrm{g}}$ & 5) & $2 a$ & 73 & $67: 33$ \\
\hline
\end{tabular}

${ }^{a}$ Unless otherwise indicated, reactions were conducted with 1.0 equiv. of substrates, 1.2 equiv. of nucleophile and $1.0 \mathrm{~mol} \%$ of catalyst in toluene $(0.1 \mathrm{M})$. Er was determined by $\mathrm{HPLC}$ and $\mathrm{GC}$. ${ }^{\mathrm{b}} 1.0 \mathrm{mmol}$ scale, $0.1 \mathrm{~mol} \%$ catalyst. ${ }^{\mathrm{c}} 0.3 \mathrm{~mol} \%$ catalyst. ${ }^{\mathrm{d}} 0.5 \mathrm{M}$. ${ }^{\mathrm{e}} 7 \mathrm{~d}$. f 4 d. ${ }^{g} 10 \mathrm{~min}$.
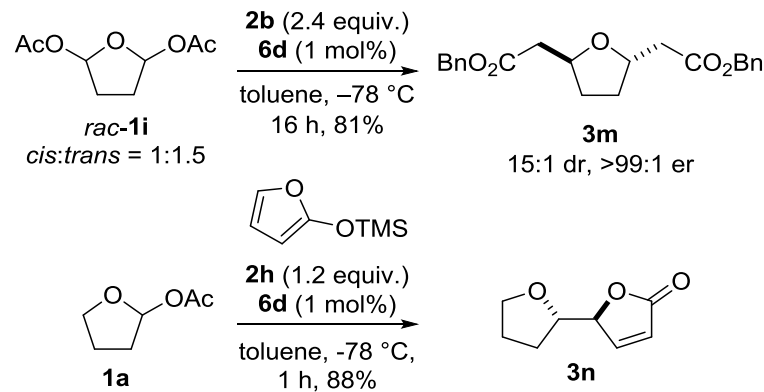

$3 n$

dr 3:1, er 98:2 cis:trans $=1: 1.5$

Towards elucidating the mechanism of our reaction, we have determined enantiomeric excesses of substrate 1a and product $\mathbf{3} \mathbf{a}$ during the course of the reaction. While the enantiomeric excess of the product was constantly high from the beginning of the reaction, not unexpectedly, one enantiomer of substrate 1a reacted faster than the other, affording a gradual enrichment of enantiomeric excess of the substrate. This phenomenon well corresponds to a theoretical graph using the equation of Kagan, ${ }^{19}$ with a selectivity factor $s$ of ca. 4 . Both, the decrement of reaction rate by electron deficiency on oxygen (Table 2 , entries 10 , 11 ) and the fact that both enantiomers are converted into the same enantiopure product are consistent with an $S_{N} 1$ mechanism involving a cyclic oxocarbenium ion intermediate paired with the chiral counteranion.

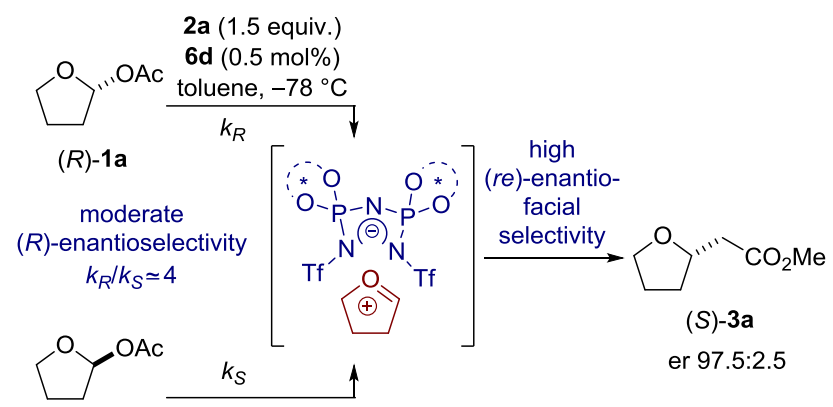

$(S)-1 \mathbf{a}$

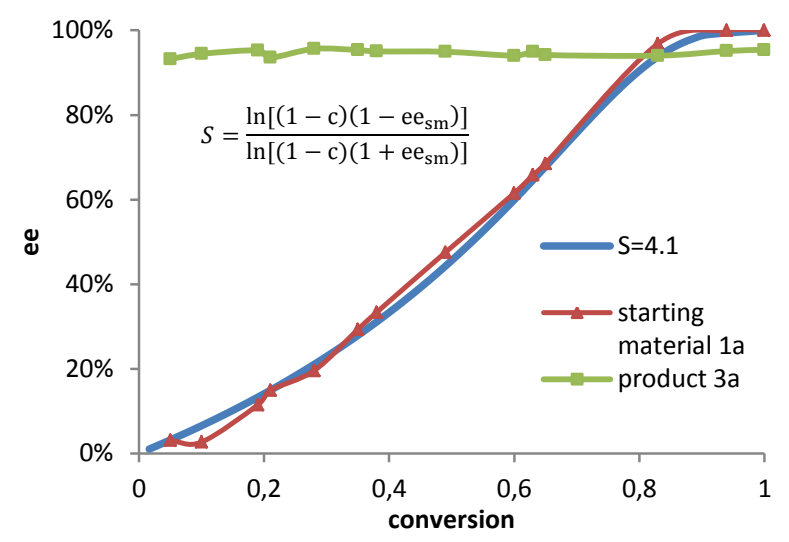

Figure 1. Proposed Mechanism and Enantiomeric Excess Monitoring Over Conversion 
In conclusion, we have developed a catalytic enantioselective synthesis of oxygen containing heterocycles from readily accessible lactol acetate precursors and silylated nucleophiles. We suggest the reaction to proceed via an ACDCtype $S_{N} 1$-mechanism involving a non-stabilized, aliphatic, cyclic oxocarbenium ion paired to the confined IDPi counteranion. The scope of our reaction is broad, and five, six, and seven-membered ring lactol acetates react with various nucleophiles. Moreover, our catalyst system also tolerates aromatic substrates furnishing chroman and dihydrobenzofuran products. Finally, a highly diastereo- and enantioconvergent catalytic asymmetric transformation has been realized with substrate $\mathbf{1 h}$ and provided a $C_{2}$-symmetric tetrahydrofuran product.

\section{ASSOCIATED CONTENT}

\section{Supporting Information}

Experimental procedures, characterizations and analytical data of products, and spectra of NMR and HPLC. This material is available free of charge via the Internet at http://pubs.acs.org.

\section{AUTHOR INFORMATION}

\section{Corresponding Author}

*list@kofo.mpg.de

\section{Notes}

The authors declare no competing financial interest.

\section{ACKNOWLEDGMENT}

Generous support by the Max-Planck-Society and the European Research Council (Advanced Grant "CHAOS") is gratefully acknowledged. We thank Roberta Properzi and Lucas Schreyer for their contributions and the members of our NMR, MS, HPLC, and GC departments for their excellent service.

\section{REFERENCES} 599.

(1) Krasnova, L.; Wong, C. H. Annu. Rev. Biochem 2016, 85,

(2) (a) Braun, M.; Kotter, W. Angew. Chem. Int. Ed. 2004, 43 514; (b) Reisman, S. E.; Doyle, A. G.; Jacobsen, E. N. J. Am. Chem. Soc. 2008, 130, 7198; (c) Benfatti, F.; Benedetto, E.; Cozzi, P. G. Chem. Asian. J. 2010, 5, 2047; (d) Moquist, P. N.; Kodama, T.; Schaus, S. E. Angew. Chem. Int. Ed. 2010, 49, 7096; (e) Burns, N. Z.; Witten, M. R.; Jacobsen, E. N. J. Am. Chem. Soc. 2011, 133, 14578; (f) Maity, P.; Srinivas, H. D.; Watson, M. P. J. Am. Chem. Soc. 2011, 133, 17142; (g) Rueping, M.; Volla, C. M. R.; Atodiresei, I. Org. Lett. 2012, 14, 4642; (h) Watson, M. P.; Maity, P. Synlett 2012, 23, 1705; (i) Cui, Y.; Villafane, L. A.; Clausen, D. J.; Floreancig, P. E. Tetrahedron 2013, 69, 7618; (j) Hsiao, C.-C.; Liao, H.-H.; Sugiono, E.; Atodiresei, I.; Rueping, M. Chem. Eur. J. 2013, 19, 9775; (k) Terada, M.; Yamanaka, T.; Toda, Y. Chem. Eur. J. 2013, 19, 13658; (I) Meng, Z.; Sun, S.; Yuan, H.; Lou, H.; Liu, L. Angew. Chem. Int. Ed. 2014, 53, 543; (m) Terada, M.; Li, F.; Toda, Y. Angew. Chem. Int. Ed. 2014, 53, 235; (n) Dasgupta, S.; Rivas, T.; Watson, M. P. Angew. Chem. Int. Ed. 2015, 54, 14154; (o) Srinivas, H. D.; Maity, P.; Yap, G. P. A.; Watson, M. P. J. Org. Chem. 2015 80, 4003; (p) Hardman-Baldwin, A. M.; Visco, M. D.; Wieting, J. M.; Stern, C.; Kondo, S.-i.; Mattson, A. E. Org. Lett. 2016, 18, 3766; (q) Yang, Z.; He, Y.; Toste, F. D. J. Am. Chem. Soc. 2016, 138, 9775. (r) Ford, D. D.; Lehnherr, D.; Kennedy, C. R.; Jacobsen, E. N. ACS Catal. 2016, 6, 4616; (s) Ford, D. D.; Lehnherr, D.; Kennedy, C. R.; Jacobsen, E. N. J. Am. Chem. Soc. 2016, 138, 7860; (t) Kennedy, C. R.; Lehnherr, D.; Rajapaksa, N. S.; Ford, D. D.; Park, Y.; Jacobsen, E. N. J. Am. Chem. Soc. 2016, 138, 13525.

(3) (a) Faul, M. M.; Huff, B. E. Chem. Rev. 2000, 100, 2407; (b) Kang, E. J.; Lee, E. Chem. Rev. 2005, 105, 4348.

(4) (a) Wolfe, J. P.; Hay, M. B. Tetrahedron 2007, 63, 261; (b) Jalce, G.; Franck, X.; Figadère, B. Tetrahedron: Asymmetry 2009, 20, 2537.

(5) Haloetherification: (a) Kang, S. H.; Lee, S. B.; Park, C. M. J. Am. Chem. Soc. 2003, 125, 15748; (b) Hennecke, U.; Müller, C. H.; Fröhlich, R. Org. Lett. 2011, 13, 860; (c) Denmark, S. E.; Burk, M. T. Org. Lett. 2012, 14, 256; (d) Miles, D. H.; Veguillas, M.; Toste, F. D. Chem. Sci. 2013, 4, 3427; (e) Zeng, X.; Miao, C.; Wang, S.; Xia, C.; Sun, W. Chem. Commun. 2013, 49, 2418; (f) Ke, Z.; Tan, C. K.; Chen, F.; Yeung, Y.-Y. J. Am. Chem. Soc. 2014, 136, 5627; (g) Müller, C. H.; Rösner, C.; Hennecke, U. Chem. Asian. J . 2014, 9, 2162; (h) Tay, D. W.; Leung, G. Y. C.; Yeung, Y.-Y. Angew. Chem. Int. Ed. Engl. 2014, 53, 5161.; (i) Ke, Z.; Tan, C. K.; Liu, Y.; Lee, K. G. Z.; Yeung, Y.-Y. Tetrahedron 2016, 72, 2683.

(6) Epoxide ring opening: (a) Blanc, A.; Toste, F. D. Angew. Chem. Int. Ed. 2006, 45, 2096; (b) Jensen, K. H.; Pathak, T. P.; Zhang, Y.; Sigman, M. S. J. Am. Chem. Soc. 2009, 131, 17074.

(7) Oxy-Michael addition reaction: (a) Asano, K.; Matsubara, S. J. Am. Chem. Soc. 2011, 133, 16711; (b) Nising, C. F.; Bräse, S. Chem. Soc. Rev. 2012, 41, 988; (c) Cox, N.; Uehling, M. R.; Haelsig, K. T.; Lalic, G. Angew. Chem. Int. Ed. 2013, 52, 4878; (d) Hintermann, L.; Ackerstaff, J.; Boeck, F. Chem. Eur. J. 2013, 19, 2311; (e) Kobayashi, Y.; Taniguchi, Y.; Hayama, N.; Inokuma, T.; Takemoto, Y. Angew. Chem. 2013, 125, 11320; (f) Lu, Y.; Zou, G.; Zhao, G. ACS Catalysis 2013, 3, 1356; (g) Azuma, T.; Murata, A.; Kobayashi, Y.; Inokuma, T.; Takemoto, Y. Org. Lett. 2014, 16, 4256; (h) Miyaji, R.; Asano, K.; Matsubara, S. Org. Biomol. Chem. 2014, 12, 119; (i) Ravindra, B.; Das, B. G.; Ghorai, P. Org. Lett. 2014, 16, 5580; (j) Ravindra, B.; Maity, S.; Das, B. G.; Ghorai, P. J. Org. Chem. 2015, 80, 7008.

(8) Hydroformylation: (a) Adint, T. T.; Wong, G. W.; Landis, C. R. J. Org. Chem. 2013, 78, 4231; (b) Xu, K.; Zheng, X.; Wang, Z.; Zhang, X. Chem. Eur. J. 2014, 20, 4357.

(9) Metal catalyzed alkoxylation: (a) Hosokawa, T.; Uno, T.; Inui, S.; Murahashi, S. J. Am. Chem. Soc. 1981, 103, 2318; (b) Uozumi, Y.; Kato, K.; Hayashi, T. J. Am. Chem. Soc. 1997, 119, 5063; (c) Trend, R. M.; Ramtohul, Y. K.; Ferreira, E. M.; Stoltz, B. M. Angew. Chem. Int. Ed. 2003, 42, 2892; (d) Zhang, Z.; Widenhoefer, R. A. Angew. Chem. Int. Ed. 2007, 46, 283.

(10) C-H bond activation Davies, H. M. L.; Hansen, T.; Churchill, M. R. J. Am. Chem. Soc. 2000, 122, 3063.

(11) Prins cyclization: (a) Lalli, C.; van de Weghe, P. Chem. Commun. 2014, 50, 7495; (b) Liu, J.; Zhou, L.; Wang, C.; Liang, D.; Li, Z.; Zou, Y.; Wang, Q.; Goeke, A. Chem. Eur. J. 2016, 22, 6258; (c) Liu, L.; Kaib, P. S. J.; Tap, A.; List, B. J. Am. Chem. Soc. 2016, 138, 10822.

(12) (a) Čorić, I.; List, B. Nature 2012, 483, 315; (b) Liu, L.; Leutzsch, M.; Zheng, Y.; Alachraf, M. W.; Thiel, W.; List, B. J. Am. Chem. Soc. 2015, 137, 13268.

(13) (a) Kaib, P. S. J.; Schreyer, L.; Lee, S.; Properzi, R.; List, B. Angew. Chem. Int. Ed. 2016, 55, 13200; (b) Xie, Y.; Cheng, G. J.; Lee, S.; Kaib, P. S. J.; Thiel, W.; List, B. J. Am. Chem. Soc. 2016, $138,14538$.

(14) (a) García-García, P.; Lay, F.; García-García, P.; Rabalakos, C.; List, B. Angew. Chem. Int. Ed. 2009, 48, 4363; (b) Tap, A.; Blond, A.; Wakchaure, V. N.; List, B. Angew. Chem. Int. Ed. 2016, 55, 8962; (c) Zhang, Z.; Bae, H. Y.; Guin, J.; Rabalakos, C.; van Gemmeren, M.; Leutzsch, M.; Klussmann, M.; List, B. Nat. Commun. 2016, 7, 12478; for review: (d) James, T.; van Gemmeren, M.; List, B. Chem. Rev. 2015, 115, 9388;

(15) Mahlau, M.; List, B. Angew. Chem. Int. Ed. 2013, 52, 518.

(16) For a review on silylium-based Lewis acid catalysis, see: (a) Dilman, A. D.; loffe, S. L. Chem. Rev. 2003, 103, 733. For nonenantioselective versions, see: (b) Murata, S.; Suzuki, M.; Noyori, R. Tetrahedron 1988, 44, 4259. (c) Larsen, C. H.; Ridgway, B. H. Shaw, J. T.; Smith, D. M.; Woerpel, K. A. J. Am. Chem. Soc. 2005 127, 10879; (d) Larsen, C. H.; Ridgway, B. H.; Shaw, J. T.; Woerpel, K. A. J. Am. Chem. Soc. 1999, 121, 12208; (e) Schmitt, A.; 
Reißig, H.-U. Eur. J. Org. Chem. 2000, 3893; (f) Shenoy, S. R.; Smith, D. M.; Woerpel, K. A. J. Am. Chem. Soc. 2006, 128, 8671.

(17) (a) Bellur, E.; Freifeld, I.; Böttcher, D.; Bornscheuer, U. T.; Langer, P. Tetrahedron 2006, 62, 7132; (b) Bloch, R.; Bortolussi, M.; Girard, C.; Seck, M. Tetrahedron 1992, 48, 453; (c) Cere, V.; Mazzini, C.; Paolucci, C.; Pollicino, S.; Fava, A. J. Org. Chem. 1993, 58, 4567; (d) Laxmi, Y. R. S.; lyengar, D. S. Synthesis 1996 594; (e) Paolucci, C.; Mazzini, C.; Fava, A. J. Org. Chem. 1995, 60, 169.

(18) See the supporting information for additional examples

(19) Kagan, H. B.; Fiaud, J. C. In Topics in Stereochemistry; Eliel, E. L., Wilen, S. H., Eds.; John Wiley \& Sons: New York, 1988; Vol. 18, pp 249-330. 